\title{
On the Periodicity of a Max-type Fuzzy Difference Equations
}

\author{
Changyou Wang ${ }^{1,2}$,, Wei Wei ${ }^{1}$, Qiang Yang ${ }^{2}$, Yonghong $\mathbf{L i}^{1}$ \\ ${ }^{1}$ College of Science, Chongqing University of Posts and Telecommunications, Chongqing, P. R. China \\ ${ }^{2}$ College of Applied Mathematics, Chengdu University of Information Technology, Chengdu, P. R. China
}

\section{Email address:}

wangchangyou417@163.com (Changyou Wang),853553304@qq.com (Wei Wei),805379650@qq.com (Qiang Yang), liyh1972@yeah.net (Yonghong Li)

*Corresponding author

\section{To cite this article:}

Changyou Wang, Wei Wei, Qiang Yang, Yonghong Li. On the Periodicity of a Max-type Fuzzy Difference Equations. American Journal of Electromagnetics and Applications. Vol. 7, No. 2, 2019, pp. 13-18. doi: 10.11648/j.ajea.20190702.11

Received: November 17, 2019; Accepted: December 2, 2019; Published: December 11, 2019

\begin{abstract}
Our aim in this paper is to discuss the periodicity and boundedness of a max-type fuzzy difference equation. When studying the periodicity of the solution to the max-fuzzy difference equation, the equation is first converted into a difference system composed of two related difference equations through the cut set theory of the fuzzy number, then the periodicity of each solution sequence in the system is obtained by means of inequality technique, mathematical induction and other theoretical methods, thus the periodicity of the solution is proved. As researching the boundedness of the solution for the fuzzy difference equation, the difference system is also obtained through the cut set theory of the fuzzy number, then analyze the boundedness to each solution sequence according to the periodicity with the solution sequence, through examining the value of the finite subsequence in each solution sequence, the boundedness with these subsequences can be obtained, and then the boundedness for each solution sequence made up of complete subsequences can be known, thus the boundedness of the solution is proved. Finally, the results obtained in this paper are simulated by using the software package MATLAB 2016, the numerical results not only show the dynamic behavior of the solutions to the fuzzy difference systems, but also verify the effectiveness of the theoretical results.
\end{abstract}

Keywords: Fuzzy Difference Equation, Max-Type, Cut Theory, Periodicity, Boundedness

\section{Introduction}

The difference equation is a kind of mathematical model describing discrete dynamic system. As an important branch of the dynamic system, the difference equation theory is widely used in population statistics and analysis, economic finance, control science, communication science and computer science (see, e.g., [1-5] and the reference therein). With the progress and development of science and technology, the difference equation will become a more and more popular research topic. As an important part of the difference equation, the max-type difference equation has important applications in many fields, such as models of automatic control theory and so on (see, e.g., [6-10] and thereference therein). Because of this, scholars have studied many theories and applications of the max-type difference equation in recent years. Since periodical research of differential equation theory has been very mature and perfect, some scholars try to investigate the periodicity of difference equation with the theory of differential equation (see, e.g., [11-17] and the reference therein).

Making a historical flash back for the equation we study in this paper, we need to mention that in 2006, Yang et al. [18] studied the following second order max-type difference equation with index parameters

$$
x_{n}=\max \left\{\frac{1}{x_{n-1}^{\alpha}}, \frac{A}{x_{n-2}}\right\}, n \in N_{0}
$$

where the parameters $A>0, \alpha \in(0,1)$, the author proved that the positive solution of the equation (1) converges to 1 when the parameter $A \leq 1$, and the positive solution of the equation (1) tends to four-periodic solution when the parameter $A>1$.

At the same time we could see that in 2012, S. Stevic [19] studied the periodicity of the solutions for the difference equations (2), 


$$
\left\{\begin{array}{c}
x_{n}^{(1)}=\max _{1 \leq i \leq m_{1}}\left\{f_{1 i}\left(x_{n-k_{i, 1}^{(1)}}^{(1)}, x_{n-k_{i, 2}^{(1)}}^{(2)}, \cdots, x_{n-k_{i, l}^{(1)}}^{(l)}, n\right), x_{n-s}^{(1)}\right\} \\
x_{n}^{(2)}=\max _{1 \leq i \leq m_{1}}\left\{f_{2 i}\left(x_{n-k_{i, 1}^{(1)}}^{(1)}, x_{n-k_{i, 2}^{(1)}}^{(1)}, \cdots, x_{n-k_{i, l}^{(1)}}^{(l)}, n\right), x_{n-s}^{(2)}\right\} \\
\vdots \\
x_{n}^{(l)}=\max _{1 \leq i \leq m_{1}}\left\{f_{l i}\left(x_{n-k_{i, 1}^{(1)}}^{(1)}, x_{n-k_{i, 2}^{(1)}}^{(2)}, \cdots, x_{n-k_{i, l}^{(1)}}^{(l)}, n\right), x_{n-s}^{(l)}\right\},
\end{array}\right.
$$

where $\quad n, s, l, m_{j}, k_{i, t}^{(j)} \in N, j, t \in\{1, \cdots, l\} \quad, \quad$ and $\quad j \in$ $\{1, \cdots, l\}, i \in\left\{1, \cdots, m_{j}\right\}$. When the parameters $j, i \in$ $\left\{1, \cdots, m_{j}\right\}$ are permanent, then the final period value of the solutions to the equations (2) is $s$. In addition, S. Stevic also dissected the periodicity of the min-type difference equations corresponding to the equations (2).

In addition, in 2002, Voulov [20] analyzed the periodicity of the positive solution of the max-type difference equation (3),

$$
x_{n}=\max \left\{\frac{A}{x_{n-k}}, \frac{B}{x_{n-m}}\right\}, n=0,1, \cdots
$$

Where $k, m$ are arbitrary positive integers, the parameters $A, B$ and the initial conditions $x_{-1}, x_{-2}, \cdots, x_{-d}$ are arbitrary positive real numbers, in which $d=\max \{k, m\}$. The periodicity of the solution for the equation is proved by the thought of classified discussions.

Recently, many scholars have further expanded the form of the equation (3). In 2009, Ali Gelisken [21] resolved the dynamic properties of the solution to the max-type difference equation (4),

$$
x_{n}=\max \left\{\frac{A}{x_{n-p}^{\alpha}}, \frac{B}{x_{n-k}^{\beta}}\right\}, n=0,1, \cdots
$$

where $p, k$ are positive integers, $0<\alpha, \beta<1$ and $A, B>0$. In this paper, the author obtained that every positive solution converges to $\bar{x}=\max \left\{A^{1 /(1+\alpha)}, B^{1 /(1+\beta)}\right\}$.

Besides, in 1999, Szalkai [22] presented the periodicity of the solution for the max-type difference equation (5),

$$
x_{n+1}=\max \left\{\frac{A}{x_{n}}, \frac{A}{x_{n-1}}, \cdots, \frac{A}{x_{n-k}}\right\}, n=0,1, \cdots
$$

where $k$ is a positive integer, the parameters $A$ and the initial conditions $\left\{x_{i}\right\}_{i=-k}^{0}$ are real numbers. In this paper, the author proved that every positive solution of the equation is periodic with $k+2$.

With the sustainable development of the max-type difference equation theory, scholars have combined it with the fuzzy sets theory (see, e.g., [23-30] and the reference therein), thus creating the max-type fuzzy difference equation, and at the same time doing numerous studies on the max-type fuzzy difference equation. In 2004, Stefanidou [31] generalized the difference equation (5) from the real number range to the fuzzy number range, that is, the parameter $A$ and the initial values $\left\{x_{i}\right\}_{i=-k}^{0}$ are positive fuzzy numbers, then the solutions of the equation are also positive fuzzy numbers. In this paper, the author gave the corresponding conditions for the solution of the equation to be periodic, unbounded and unsustainable. Meanwhile, the dynamic properties of the max-type fuzzy difference equation (6), a special form of the equation (3), are also discussed in reference [31]

$$
x_{n+1}=\max \left\{\frac{A_{0}}{x_{n}}, \frac{A_{1}}{x_{n-1}}\right\}, n=0,1, \cdots,
$$

where the parameter $A_{0}, A_{1}$ and the initial values $x_{-1}, x_{0}$ are positive fuzzy numbers given arbitrarily. In this paper, the author obtained that the solution of the equation (6) is periodic, unbounded and non-persistent. In 2006, Stefanidou [32] further extended the form of the equation (6), and considered the periodicity of the solution for the max-type type fuzzy difference equation (7),

$$
x_{n+1}=\max \left\{\frac{A_{0}}{x_{n-k}}, \frac{A_{1}}{x_{n-m}}\right\}, n=0,1, \cdots,
$$

where the parameter $A_{0}, A_{1}$ and the initial values $x_{-d}, \cdots, x_{0}, d=\max \{k, m\}$ are positive fuzzy numbers given arbitrarily.

Inspired by the discussions above, in this paper, we will study the periodic property of the solutions to the following fuzzy difference equation (8)

$$
x_{n+1}=\max \left\{\frac{A}{x_{n}}, \frac{A}{x_{n-1}}, \cdots, \frac{A}{x_{n-(k-1)}}, x_{n-k}\right\}, n=0,1,2, \cdots,
$$

where $k$ is a positive integer, the parameters $A$ and the initial conditions $x_{-k}, x_{-k+1}, \cdots, x_{0}$ are positive fuzzy numbers.

\section{Preliminaries and Notations}

For the convenience of readers, we need to give the following notations, definitions and preliminary results, see [33-37].

Definition 2.1 For a set $B$ we denote by $\bar{B}$ the closure of $B$, we say that a function $A: R \rightarrow[0,1]$ is a fuzzy number if the follow conclusions are true,

(1) $A$ is normal, i.e., there exists $x \in R$ such that $A(x)=1$;

(2) $A$ is a fuzzy convex set, i.e.,

$A\left(t x_{1}+(1-t) x_{2}\right) \geq \min \left\{A\left(x_{1}\right), A\left(x_{2}\right)\right\}, \forall t \in[0,1], x_{1}, x_{2} \in R ;$

(3) $A$ is upper semicontinuous on $R$;

(4) The support of $A$, i.e., $\operatorname{supp} A=\overline{\cup_{\alpha \in(0,1]}[A]_{\alpha}}=$ $\overline{\{x \in R: A(x)>0\}}$ is compact.

where the $\alpha$-cuts of $A$ are closed intervals, define as $[A]_{\alpha}=\{x \in R: A(x) \geq \alpha\}$. if supp $A \subset(0,+\infty)$, then fuzzy number $A$ is obviously positive.

Definition 2.2 Let $A, B$ be fuzzy numbers which are satisfying $\quad[A]_{\alpha}=\left[A_{l, \alpha}, A_{r, \alpha}\right],[B]_{\alpha}=\left[B_{l, \alpha}, B_{r, \alpha}\right]$, 
$\alpha \in(0,1]$, we denote the following metric

$$
D(A, B)=\sup \max \left\{\left|A_{l, \alpha}-B_{l, \alpha}\right|,\left|A_{r, \alpha}-B_{r, \alpha}\right|\right\}
$$

where sup is taken for all $\alpha \in(0,1]$. Then $\left(R_{f}, D\right)$ is a complete metric space. For the convenience of application in the future, we define $\hat{0} \in R_{f}$ as

$$
\hat{0}(x)=\left\{\begin{array}{l}
1, x=0 \\
0, x \neq 0
\end{array}\right.
$$

Thus, $[\hat{0}]_{\alpha}=[0,0], 0<\alpha \leq 1$.

Considering the following difference equation

$$
x_{n+1}=f\left(x_{n}, x_{n-1}, \cdots, x_{n-k}\right), n=0,1,2, \cdots
$$

where $I_{x}$ is a real number interval and $f: I_{x}^{k+1} \rightarrow I_{x}$ is a continuous function.

$$
\begin{aligned}
& x_{n+1}=f\left(x_{n}, x_{n-1}, \cdots, x_{n-k}, y_{n}, y_{n-1}, \cdots, y_{n-k}\right), n=0,1,2, \cdots, \\
& y_{n+1}=g\left(x_{n}, x_{n-1}, \cdots, x_{n-k}, y_{n}, y_{n-1}, \cdots, y_{n-k}\right), n=0,1,2, \cdots,(10)
\end{aligned}
$$

where $I_{x}, I_{y}$ are real number intervals and $f$ : $I_{x}^{k+1} \times I_{y}^{k+1} \rightarrow I_{x}, g: I_{x}^{k+1} \times I_{y}^{k+1} \rightarrow I_{y} \quad$ are continuous functions.

Definition 2.3 For the difference equation (9), the periodicity is defined as follows

(1) A sequence $\left\{x_{n}\right\}_{n=0}^{\infty}$ is called $p$-period, where $p$ is a positive integer, for all $n \in N$, we have $x_{n+p}=x_{n}$;

(2) A sequence $\left\{x_{n}\right\}_{n=0}^{\infty}$ is called the min $p$-period, for all $n \in N$, we have $x_{n+p}=x_{n}$, and $p$ is the smallest positive integer that holds this formula.

Definition 2.4 For the difference system (10), the periodicity is defined as follows

(1) If $x_{n}=x_{n+p}, y_{n}=y_{n+p}$ are satisfied for any $n \in N$, where $p$ is a positive integer, then it is said that the solutions $\left\{\left(x_{i}, y_{i}\right)\right\}_{i=-k}^{+\infty}$ of the difference system (10) are periodic with $p$.

(2) If $x_{n}=x_{n+p}, y_{n}=y_{n+p}$ are satisfied for any $n \in N$, at the same time $p$ is the smallest positive integer satisfying the difference system (10), then it is said that the solutions $\left\{\left(x_{i}, y_{i}\right)\right\}_{i=-k}^{+\infty}$ of the system $(10)$ are the min periodic with $p$.

(3) If every solution of the difference system (10) is periodic with $p$, where $p$ is a positive integer, then the solution of the system (10) is called $p$-period.

Definition 2.5 Let $\left\{x_{n}\right\}$ be a sequence of fuzzy numbers, then

(1) If there is a positive real number $M$ (or $N$ ) that holds supp $x_{n} \in(M,+\infty]\left(\right.$ or supp $\left.x_{n} \in(0, N]\right), n=0,1,2, \cdots$, then the sequence $\left\{x_{n}\right\}$ of fuzzy numbers is persistent (or bounded);
(2) If there are two positive real numbers $M, N$ satisfy supp $x_{n} \in(M, N], \quad n=0,1,2, \cdots$, then the sequence $\left\{x_{n}\right\}$ of fuzzy numbers is both persistent and bounded.

\section{Main Results and Proofs}

According to $\alpha$ - cut set theory, the max-type fuzzy difference equation (8) can be transformed into the following ordinary difference system consisting of two ordinary difference equations

$$
\begin{aligned}
& y_{n+1}=\max \left\{\frac{B}{z_{n}}, \frac{B}{z_{n-1}}, \cdots, \frac{B}{z_{n-(k-1)}}, y_{n-k}\right\}, n=0,1,2, \cdots, \\
& z_{n+1}=\max \left\{\frac{C}{y_{n}}, \frac{C}{y_{n-1}}, \cdots, \frac{C}{y_{n-(k-1)}}, z_{n-k}\right\}, n=0,1,2, \cdots,
\end{aligned}
$$

where $B, C, y_{-k}, y_{-k+1}, \cdots, y_{0}, z_{-k}, z_{-k+1}, \cdots, z_{0} \in(0,+\infty)$.

Theorem 3.1 For the difference system (11), each solution sequence $\left\{y_{n}\right\},\left\{z_{n}\right\}$ has periodicity, and the period is $(k+1)$.

Proof: According to the difference system (11), we can gain the following several inequalities

$$
y_{n} \geq y_{n-(k+1)}, z_{n} \geq z_{n-(k+1)}, y_{n+1} \geq \frac{B}{z_{n-i}},(i=0,1, \cdots,(k-1))
$$

By the synthesis method, we can deduce

$y_{n} \geq \frac{B}{z_{n-(i+1)}}, y_{n-k} \geq \frac{B}{z_{n-(i+k+1)}}=\frac{B}{z_{n-i-(k+1)}} \geq \frac{B}{z_{n-i}},(i=0,1, \cdots,(k-1))$

The inequalities above reflect the size relations of each algebra contained in the right-hand formula to an equation in the difference system (11), then through the value of each algebra relationship and definition of the equation in the system (11), we can obtain

$$
y_{n+1}=y_{n-k} \text {, Namely } y_{n}=y_{n-(k+1)}
$$

Therefore the solution sequence $\left\{y_{n}\right\}$ has periodicity, and the period is $(k+1)$.

And once again, according to the difference system (11), the following some inequalities can be obtained

$$
y_{n} \geq y_{n-(k+1)}, z_{n} \geq z_{n-(k+1)}, z_{n+1} \geq \frac{C}{y_{n-i}},(i=0,1, \cdots,(k-1))
$$

From the synthesis, we can get the following inequalities

$z_{n} \geq \frac{C}{y_{n-(i+1)}}, z_{n-k} \geq \frac{C}{y_{n-(i+k+1)}}=\frac{C}{y_{n-i-(k+1)}} \geq \frac{C}{y_{n-i}},(i=0,1, \cdots,(k-1))$

The inequalities above reflect the size relations of the algebraic expressions on the right-hand side of another equation in the difference system (11), then through the value of the size relationship and the definition of the equation in the system (11), we can gain 


$$
z_{n+1}=z_{n-k}, \text { Namely } z_{n}=z_{n-(k+1)}
$$

Thereby, the solution sequence $\left\{z_{n}\right\}$ has periodicity, and the period is $(k+1)$. The proof is completed.

Theorem 3.2 For the difference system (11), if the parameters $B, C$ and the initial conditions $y_{-k}, y_{-k+1}$, $\cdots, y_{0}, z_{-k}, z_{-k+1}, \cdots, z_{0}$ are positive real numbers, then the solution sequence $\left\{y_{n}\right\},\left\{z_{n}\right\}$ of the system (11) are bounded and persistent.

Proof: Theorem 3.1 indicates that the value of each term in the solution sequence $\left\{y_{n}\right\}$ must be one of the following values

(A) $y_{(k+1) n}=y_{0}$,

$\left(\mathrm{A}_{2}\right) y_{(k+1) n+1}=y_{1}=\max \left\{\frac{B}{z_{0}}, \frac{B}{z_{-1}}, \cdots, \frac{B}{z_{-k+1}}, y_{-k}\right\}$,

(A) $y_{(k+1) n+2}=y_{2}=\max \left\{\frac{B}{z_{1}}, \frac{B}{z_{0}}, \cdots, \frac{B}{z_{-k+2}}, y_{-k+1}\right\}=$

$\max \left\{\min \left\{\frac{B y_{0}}{C}, \frac{B y_{-1}}{C}, \cdots, \frac{B y_{-k+1}}{C}, \frac{B}{z_{-k}}\right\}, \frac{B}{z_{0}}, \cdots, \frac{B}{z_{-k+2}}, y_{-k+1}\right\}$,

(A) $y_{(k+1) n+3}=y_{3}=\max \left\{\frac{B}{z_{2}}, \frac{B}{z_{1}}, \cdots, \frac{B}{z_{-k+3}}, y_{-k+2}\right\}$,

$\left(A_{k}\right) \quad y_{(k+1) n+(k-1)}=y_{k-1}=\max \left\{\frac{B}{z_{k-2}}, \frac{B}{z_{k-3}}, \cdots, \frac{B}{z_{-1}}, y_{-2}\right\}$, $\left(\mathrm{A}_{\mathrm{k}+1}\right) y_{(k+1) n+k}=y_{k}=\max \left\{\frac{B}{z_{k-1}}, \frac{B}{z_{k-2}}, \cdots, \frac{B}{z_{0}}, y_{-1}\right\}$.

Likewise, the value of each term in the solution sequence $\left\{z_{n}\right\}$ must have one of the following values

(B) $z_{(k+1) n}=z_{0}$

$\left(\mathrm{B}_{2}\right) z_{(k+1) n+1}=z_{1}=\max \left\{\frac{C}{y_{0}}, \frac{C}{y_{-1}}, \cdots, \frac{C}{y_{-k+1}}, z_{-k}\right\}$,

(B) $z_{(k+1) n+2}=z_{2}=\max \left\{\frac{C}{y_{1}}, \frac{C}{y_{0}}, \cdots, \frac{C}{y_{-k+2}}, z_{-k+1}\right\}=$ $\max \left\{\min \left\{\frac{C z_{0}}{B}, \frac{C z_{-1}}{B}, \cdots, \frac{C z_{-k+1}}{B}, \frac{C}{y_{-k}}\right\}, \frac{C}{y_{0}}, \cdots, \frac{C}{y_{-k+2}}, z_{-k+1}\right\}$,

(B) $z_{(k+1) n+3}=z_{3}=\max \left\{\frac{C}{y_{2}}, \frac{C}{y_{1}}, \cdots, \frac{C}{y_{-k+3}}, z_{-k+2}\right\}$,

$\left(B_{k}\right) \quad z_{(k+1) n+(k-1)}=z_{k-1}=\max \left\{\frac{C}{y_{k-2}}, \frac{C}{y_{k-3}}, \cdots, \frac{C}{y_{-1}}, z_{-2}\right\}$,

$\left(\mathrm{B}_{\mathrm{k}+1}\right) z_{(k+1) n+k}=z_{k}=\max \left\{\frac{C}{y_{k-1}}, \frac{C}{y_{k-2}}, \cdots, \frac{C}{y_{0}}, z_{-1}\right\}$.

As can be seen from the equation $\left(\mathrm{B}_{3}\right), z_{2}$ is bounded and persistent, so $y_{3}$ in the equation $\left(\mathrm{A}_{4}\right)$ is bounded and persistent.

Similarly from the equation $\left(\mathrm{A}_{3}\right)$, we can see that $y_{2}$ is bounded and persistent, then we can know that $z_{3}$ in the equation $\left(\mathrm{B}_{4}\right)$ is bounded and persistent.

By induction, we can conclude that: from the equation $\left(B_{k}\right)$, we can get that $z_{k-1}$ is bounded and persistent, then we can figure out that $y_{k}$ in the equation $\left(\mathrm{A}_{\mathrm{k}+1}\right)$ is bounded and persistent.

In a similar way, from the equation $\left(\mathrm{A}_{\mathrm{k}}\right), y_{k-1}$ is bounded and persistent, so $z_{k}$ in the equation $\left(\mathrm{B}_{\mathrm{k}+1}\right)$ is bounded and persistent.

According to the mathematical complete induction, the solution sequences $\left\{y_{n}\right\},\left\{z_{n}\right\}$ of the system (11) are bounded and continuous. The proof is completed.

\section{Numerical Simulation}

In order to verify the conclusions obtained in this paper, some numerical simulations are carried out by using the software package MATLAB 2016.

Example 4.1 Considering the case of the difference system (11) at $k=4$, i.e. the following max-type fuzzy difference equation

$$
\begin{aligned}
& y_{n+1}=\max \left\{\frac{B}{z_{n}}, \frac{B}{z_{n-1}}, \frac{B}{z_{n-2}}, \frac{B}{z_{n-3}}, y_{n-4}\right\}, n=0,1,2, \cdots, \\
& z_{n+1}=\max \left\{\frac{C}{y_{n}}, \frac{C}{y_{n-1}}, \frac{C}{y_{n-2}}, \frac{C}{y_{n-3}}, z_{n-4}\right\}, n=0,1,2, \cdots,
\end{aligned}
$$

The following figure shows the simulation results of the numerical solutions for the difference system (12).

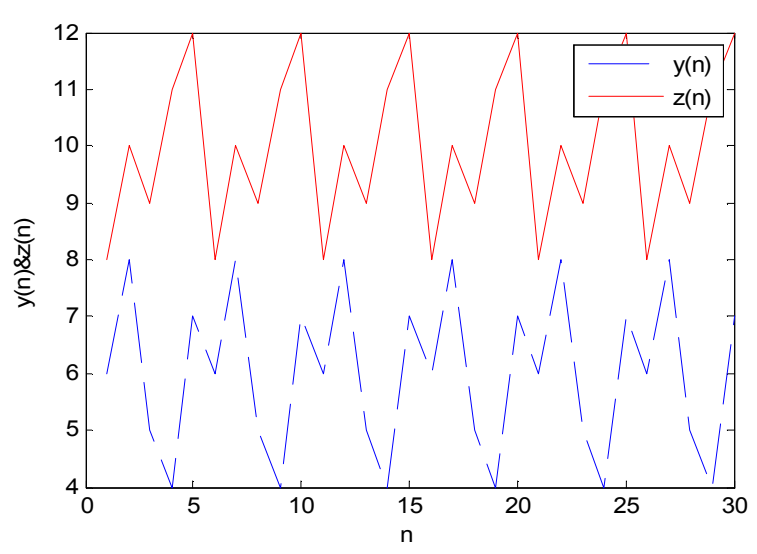

Figure 1. The numerical solutions of the difference system (12).

Figure 1. shows the numerical solutions of $\left\{y_{n}\right\},\left\{z_{n}\right\}$ for the difference system (12) when $B=5, C=9, y_{-4}=6$, $y_{-3}=8, y_{-2}=5, y_{-1}=4, y_{0}=7, z_{-4}=8, z_{-3}=10, z_{-2}=9$, $z_{-1}=11, z_{0}=12$, it can be seen from Figure 1 . that the solutions of the difference system (12) are 5-period, bounded and persistent, which verifies the conclusions of Theorem 3.1 and Theorem 3.2.

\section{Conclusion}

In this paper, we study the periodicity and boundedness of 
the solutions to a max-type difference equation. Firstly, by using iteration method, inequality skills and mathematical induction, we proved that the max-type difference equation has period of $(k+1)$ when the parameters and the initial conditions are all positive fuzzy numbers. Secondly, it is proved that the solutions for the max-type difference equation is bounded and persistent when the parameters and the initial conditions are all positive fuzzy numbers. Finally, the effectiveness of the theoretical results is verified by the numerical experiments with the software package MATLAB 2016.

\section{Acknowledgements}

This work is supported by the Sichuan Science and Technology Program (Grant no. 2018JY0480) of China, the Natural Science Foundation Project of CQ CSTC (Grant no. cstc2015jcyj BX0135) of China, the National Nature Science Fund (Project no. 11572320) of China.

\section{References}

[1] S. Ellner (1984). Asymptotic behavior of some stochastic difference equation population models. Journal of Mathematical Biology 19, 169-200.

[2] Z. Jing, B. G. Zhang (2003). The positive solutions of difference equations relevant to an economic problem. Computers \& Mathematics with Applications 45, 835-842.

[3] A. J. Avello, B. M. Al-Hadithi, M. I. G. Garcia (2012). Difference equation matrix model (DEMM) for the control of wind turbines. Wind Energy 17, 57-74.

[4] S. Papadimitriou, A. Bezerianos, T. Bountis (1997). Secure communication with chaotic systems of difference equations. IEEE Transactions on Computers 46, 27-38.

[5] D. T. Tollu, Y. Yazlik, N. Taskara (2013). On the solutions of two special types of Riccati difference equation via Fibonacci numbers. Advances in Difference Equations, Article ID: 174.

[6] I. Yalçinkaya, B. D. Iricanin, C. Çinar (2007). On a Max-Type Difference Equation. Discrete Dynamics in Nature and Society, Article ID: 47264.

[7] Y. Yazlik, D. T. Tollu, N. Taskara (2015). On the solutions of a max-type difference equation system. Mathematical Methods in the Applied Sciences 38, 4388-4410.

[8] J. L. Williams (2016). On a class of nonlinear max-type difference equations. Cogent Mathematics 3, Article ID: 1269596.

[9] X. F. Yang, W. P. Liu, J. M. Liu (2011). Global Attractivity of a Family of Max-Type Difference Equations. Discrete Dynamics in Nature and Society, Article ID: 506373.

[10] S. Stević (2013). On a symmetric system of max-type difference equations. Applied Mathematics and Computation 219, 8407-8412.

[11] E. M. Elsayed (2014). On the solutions and periodic nature of some systems of difference equations. International Journal of Biomathematics 7, Article ID: 1450067.
[12] C. Y. Wang, X. T. Jing, X. H. Hu, R. Li (2017). On the periodicity of a max-type rational difference equation. Journal of Nonlinear Sciences and Applications 10, 4648-4661.

[13] M. M. El-Dessoky (2014). On the periodicity of solutions of max-type difference equation. Mathematical Methods in the Applied Sciences 38, 3295-3307.

[14] T. X. Sun, H. J. Xi (2016). Dynamics of a max-type system of difference equations. Analysis and Mathematical Physics 6, 393-402.

[15] T. X. Sun, J. Liu, Q. L. He (2014). Eventually Periodic Solutions of a Max-Type Difference Equation. The Scientific World Journal, Article ID: 319437.

[16] S. Stević, M. A. Alghamdi, A. Alotaibi (2014). Long-term behavior of positive solutions of a system of max-type difference equations. Applied Mathematics and Computation $235,567-574$.

[17] K. S. Berenhaut, J. D. Foley, S. Stević (2006). Boundedness character of positive solutions of a max difference equation. Journal of Difference Equations and Application 12, 1193-1199.

[18] X. F. Yang, X. F. Liao, C. D. Li (2006). On a difference equation with maximum. Applied Mathematics and Computation 181, 1-5.

[19] S. Stević (2012). On some periodic system of max-type difference equations. Applied Mathematics and Computation 218, 11484-11487.

[20] H. D. Voulov (2002). On the periodic character of some difference equations. Journal of Difference Equations and Applications 8, 799-810.

[21] C. Cengiz, A. Gelisken (2009). On the Global Attractivity of a Max-Type Difference Equations. Discrete Dynamics in Nature and Society, Article ID: 812674.

[22] I. Szalkai (1999). On the periodicity of the sequence $x_{\mathrm{n}+1}=\max \left\{A / x_{n}, \quad A / x_{\mathrm{n}-1}, \quad \ldots A / x_{\mathrm{n}-\mathrm{k}}\right\}, \quad \mathrm{n}=0,1, \ldots . . \quad$ Journal of Difference Equations and Applications 5, 25-29.

[23] P. Diamond (2000). Stability and periodicity in fuzzy differential equations. IEEE Transactions on Fuzzy Systems 8, 583-590.

[24] C. Y. Wang, X. L. Su, P. Liu, X. H. Hu, R. Li (2017). On the dynamics of a five-order fuzzy difference equation. Journal of Nonlinear Sciences and Applications 10, 3303-3319.

[25] L. A. Zadeh (1965). Fuzzy sets. Information Control 8, 338-353.

[26] E. Y. Deeba, A. de Korvin (1995). On a fuzzy difference equation. IEEE Transactions on Fuzzy Systems 3, 469-473.

[27] S. P. Mondal, M. Mandal, D. Bhattacharya (2017). Non-linear interval-valued fuzzy numbers and their application in difference equations. Granular Computing 3, 177-189.

[28] A. Khastan (2017). New solutions for first order linear fuzzy difference equations. Journal of Computational and Applied Mathematics 312, 156-166.

[29] Q. H. Zhang, Z. G. Luo, J. Z. Liu, Y. F. Shao (2015). Dynamical behaviour of second-order rational fuzzy difference equation. International Journal of Dynamical Systems and Differential Equations 5, 336-353. 
[30] Q. H. Zhang, L. H. Yang, D. X. Liao (2014). On first order fuzzy Ricatti difference equation. Information Sciences 270, 226-236.

[31] G. Stefanidou, G. Papaschinopoulos (2005). Behavior of the positive solution of fuzzy max-difference equations. Advances in the Difference Equations, Article ID: 947038.

[32] G. Stefanidou, G. Papaschinopoulos (2006). The periodic nature of the positive solutions of a non-linear fuzzy max-difference equations. Information Sciences 176, 3696-3710.

[33] B. Bebe (2013). Mathematics of Fuzzy Sets and Fuzzy Logic. Study in Fuzziness and Soft Computing, Springer, Heidelberg.
[34] P. Diamond, P. Kloeden (1990). Metric spaces of fuzzy sets. Fuzzy Sets and Systems 35, 241-249.

[35] V. L. Kocic, G. Ladas (1993). Mathematics and Its Applications: Global Behavior of Nonlinear Difference Equations of Higher Order with Applications. Kluwer Academic, Dordrecht.

[36] E. Y. Deeba, A. D. Korvin, E. L. Koh (1996). A fuzzy difference equation with an application. Journal of Difference Equations and Applications 2, 365-374.

[37] V. Lakshmikantham, A. S. Vatsala (2002). Basic theory of fuzzy difference equations. Journal of Difference Equations and Applications 8, 957-968. 